\title{
Bilateral Luxatio Erecta: An Atypical Presentation at the Emergency Department
}

\author{
Miguel Quesado Diogo Soares João Afonso Daniel Lopes \\ Fernando Silva Jorge Mendes \\ Serviço de Ortopedia e Traumatologia, Centro Hospitalar do Tâmega e Sousa, Penafiel, Portugal
}

\section{Keywords}

Bilateral luxatio erecta · Bilateral inferior glenohumeral dislocation · Bilateral inferior shoulder dislocation

\begin{abstract}
Bilateral luxatio erecta remains a rare condition, with less than 30 cases currently described in the literature. The authors present a case of bilateral inferior glenohumeral dislocation after a fall with low-energy trauma, treated with closed reduction and immobilization for 3 weeks followed by a physiotherapy program for functional rehabilitation. After 1 year of follow-up, the patient presented satisfactory results with a complete recovery of the previous mobility arch of both shoulders.
\end{abstract}

\section{Introduction}

Luxatio erecta or inferior glenohumeral dislocation is a rare lesion, representing around $0.5 \%$ of shoulder dislocation [1-3]. This usually appears after the trauma of the upper limb by a mechanism of forced hyperabduction, with a typical clinical presentation consisting of abduction of the arm, flexion of the elbow, and pronation of the forearm [4,5]. Complications described as fractures and rotator cuff tears can occur in $80 \%$ of the patients, followed by neurological injury in $60 \%$ of them, highlighting the importance of early diagnosis and treatment $[1,6]$.

\section{Case Report/Case Presentation}

A 77-year-old woman, with multiple comorbidities, admitted to the emergency department after syncope and frontal fall with trauma to the upper limbs. On clinical examination, she presented both arms with $120^{\circ}$ of abduction and elbows in $30^{\circ}$ of flexion, with 


\section{Case Reports in Orthopedic Research}

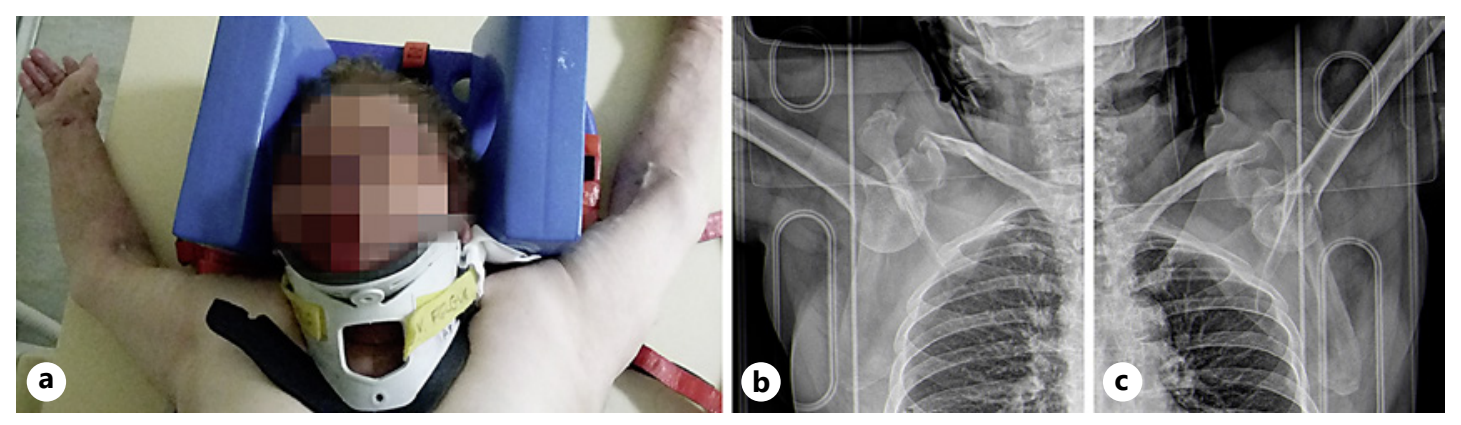

Fig. 1. Patient presentation at the emergency department (a); X-ray showing a bilateral inferior glenohumeral dislocation of the right (b) and left shoulders (c).

pain and limitation of passive or active mobilization, without neurovascular injuries. The radiograph showed bilateral inferior glenohumeral dislocation and the patient was promptly submitted to closed reduction under sedation through the traction-contraction maneuver (Fig. 1). The computed tomography scan showed small fractures without displacement of the internal proximal slope of the left humerus and the anterior wall of the right glenoid (Fig. 2). She was immobilized by bilateral slings with arms positionned in adduction and internal rotation for 3 weeks and observed in consultation, without presenting omalgia or joint stiffness, although with significant limitation of active mobility. The magnetic resonance imaging showed tendon changes compatible with partial rotator cuff tears, having started a physiotherapy program for functional rehabilitation (Fig. 3). She was reassessed 6 months later, showing an improvement in the range of mobility $\left(45^{\circ}\right.$ of flexion and $60^{\circ}$ of abduction bilaterally), despite a still significant restriction on activities of daily living (DASH score 51.7). After 1 year of follow-up, she was satisfied with a complete recovery of the shoulder mobility and no more signs of instability (bilateral abduction of $110^{\circ}$, without blocking of internal and external rotations; DASH score 10.7).

\section{Discussion/Conclusion}

The glenohumeral joint has the greatest range of motion amongst all joints in the body and, consequently, has the higher incidence of dislocations when compared to any other joint [1]. First described by Middledorpf and Scharm in 1859, lower dislocation accounts for approximately $0.5 \%$ of all cases of shoulder dislocation $[7,8]$. The likelihood that an individual can withstand the specific direction of forces to displace both shoulders simultaneously makes bilateral occurrence an extremely rare form of presentation. Murrad described the first report of bilateral presentation in 1920, currently with less than 30 cases described in the literature $[8,9]$. In the recent meta-analysis by Nambiar et al. [10], there was a prevalence of $15 \%$ of bilateral dislocation cases in a total of 199 patients with lower glenohumeral dislocation. The most common mechanism of injury, as observed in this case, comprises an indirect force of hyperabduction with a buttress effect of the humeral neck against the acromion, leading to a lever effect on the humeral head and consequent inferior dislocation [3-5]. Another mechanism is a direct trauma with axial load and inferior direction over the affected limb with an abducted arm and elbow in extension $[1,6]$. Conventional radiography with anteroposterior and axillary lateral views allows establishment of the diagnosis and exclusion of possible fractures, with computed tomography scan being reserved for further characterization. Among

\section{Karger's}




\section{Case Reports in Orthopedic Research}
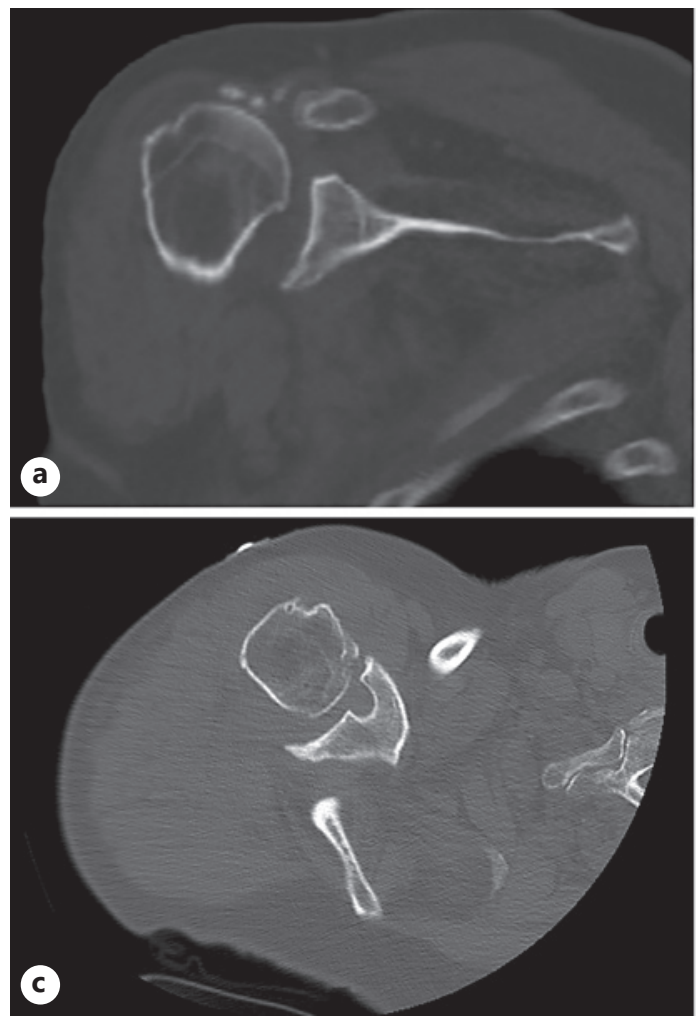
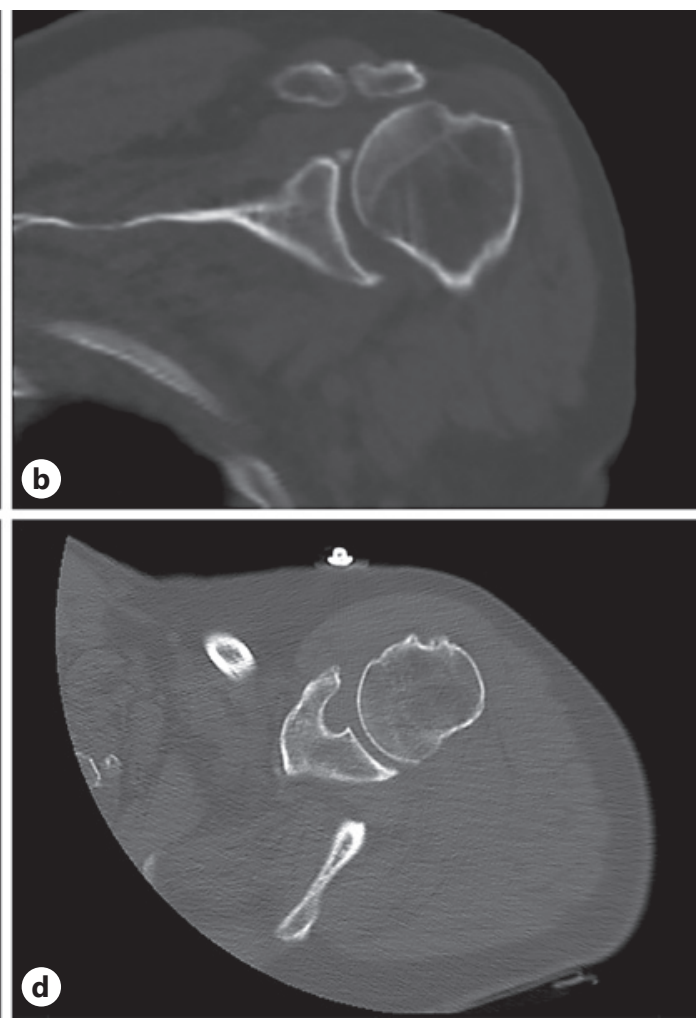

Fig. 2. CT scan of the right $(\mathbf{a}, \mathbf{c})$ and left $(\mathbf{b}, \mathbf{d})$ shoulders after close reduction by traction-contraction maneuver.

the various complications observed, fractures and rotator cuff tears remains the most frequent in $80 \%$ of cases, the last being best evaluated through magnetic resonance imaging $[1,2]$. In this case, the presence of traumatic pathology of the rotator cuff with partial tear and an undefined period of time, allowed the implementation of an effective rehabilitation protocol, resulting in gradual improvement of mobility and satisfactory functional outcomes. Neurological injuries may appear in $60 \%$ of cases, with the axillary nerve being the most frequently affected. However, these usually occur as a neuropraxia with spontaneous resolution between 2 weeks and 1 year after reduction of the dislocation [6,9]. Despite being more frequent than in other types of shoulder dislocation, vascular injury remains rare and it is described in only $3 \%$ of cases of inferior dislocation $[6,11]$. The initial treatment must be an immediate reduction of the dislocation using adequate anesthesia and muscle relaxation. Among the different reduction maneuvers suggested, the traction-contraction technique described by Freundlich, proved to be effective and safe [12]. Nho el al. also described another reduction technique involving the anterior translocation of the humeral head through smooth rotation followed by traction and replacement in the glenoid fossa [13]. After the dislocation has been reduced, the arm must be immobilized in adduction and internal rotation during 2-3 weeks [4]. Surgical treatment by open approach should be performed in cases of non-reducible dislocation with neurological injury $[6,9]$. In view of the patient's functional status and the absence of neurovascular lesions, a conservative approach was performed without the need of additional surgical intervention. Although the prognosis is usually favorable, some patients may experience chronic changes such as adhesive capsulitis, neurological injury or recurrent joint instability [14]. The authors consider it is important to publicize this lesion, in order to alert 


\section{Case Reports in Orthopedic Research}

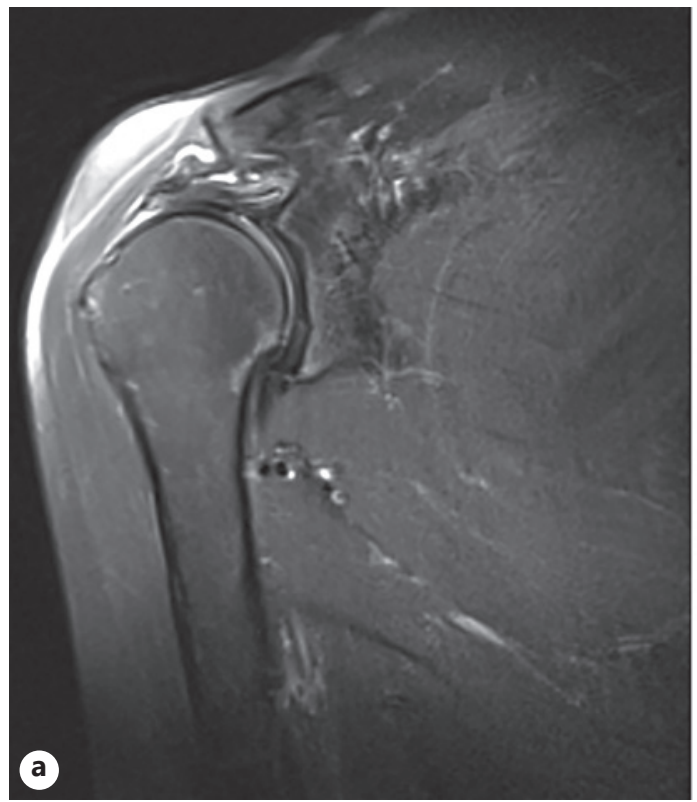

Fig. 3. MRI of the right (a) and left (b) shoulders after 3 weeks showing signs of partial rotator cuff tears.

clinicians to early diagnosis and treatment, improving the functional results and preventing secondary injuries associated with it.

\section{Statement of Ethics}

The patient provided written informed consent for the publication of this case (including images). The research was conducted ethically in accordance with the World Medical Association Declaration of Helsinki.

\section{Conflict of Interest Statement}

The authors have no conflicts of interest to declare.

\section{Funding Sources}

This research did not receive any specific funding from public, commercial, or nonprofit agencies or sectors.

\section{Author Contributions}

All the authors listed in this publication contributed substantially to the conception or design of the work, data collection, analysis, and writing the final manuscript. D. Lopes was the project administrator. 


\section{References}

1 Acosta CAX, Da Silva Resch E, Rodrigues R. Luxatio erecta bilateral: um relato de caso. Rev Bras Ortop. 2012; 47(1):130-2.

2 Lippert J, Desai B. Bilateral luxatio erecta: a case report. Case Reports Clin Med. 2018;7:608-12.

3 Tsuchida T, Yang K, Kimura Y, Taniwaki M, Ishigaki S, Itoi E. Luxatio erecta of bilateral shoulders. J Shoulder Elb Surg. 2001;10(6):595-7.

4 Camarda L, Martorana U, D’Arienzo M. A case of bilateral luxatio erecta. J Orthop Traumatol. 2009;10:97-9.

5 Petty K, Price J, Kharasch M, Novack J. Bilateral luxatio erecta: a case report. J Emerg Med. 2014;46(2):176-9.

6 Karaoglu S, Guney A, Ozturk M, Kekec Z. Bilateral luxatio erecta humeri. Arch Orthop Trauma Surg. 2003;123: 308-10.

7 Mesa M, Carpintero P, Carpintero J. Bilateral luxatio erecta humeri. Acta Orthop Belg. 1996;62(2):116-9.

8 Kumar KS, O’Rourke S, Pillay JG. Hands up: a case of bilateral inferior shoulder dislocation. Emerg Med J. 2001; 18(5):404-5.

9 Musmeci E, Gaspari D, Sandri A, Regis D, Bartolozzi P. Bilateral luxatio erecta humeri associated with a unilateral brachial plexus and bilateral rotator cuff injuries. J Orthop Trauma. 2008;22(7):498-500.

10 Nambiar M, Owen D, Moore P, Carr A, Thomas M. Traumatic inferior shoulder dislocation: a review of management and outcome. Eur J Trauma Emerg Surg. 2018;44(1):45-51.

11 Garcia R, Ponsky T, Brody F, Long J. Bilateral luxatio erecta complicated by venous thrombosis. J Trauma. 2006; 60(5):1132-4.

12 Freundlich BD. Luxatio erecta. J Trauma. 1983;23(5):434-6.

13 Nho SJ, Dodson CC, Bardzik KF, Brophy RH, Domb BG, MacGillivray JD. The two-step maneuver for closed reduction of inferior glenohumeral dislocation (luxatio erecta to anterior dislocation to reduction). J Orthop Trauma. 2006;20(5):354-7.

14 Reddy SV, Jaiswal A, Kanwar CS. A rare case of bilateral luxatio erecta with bilateral greater tuberosity fracture following a fall due to seizure. J Clin Orthop Trauma. 2019;10(3):503-6. 